\title{
Plasticity-Related PKM $\zeta$ Signaling in the Insular Cortex Is Involved in the Modulation of Neuropathic Pain after Nerve Injury
}

\author{
Jeongsoo Han, ${ }^{1,2}$ Minjee Kwon, ${ }^{1,2}$ Myeounghoon Cha, ${ }^{1}$ Motomasa Tanioka, ${ }^{1,2}$ \\ Seong-Karp Hong, ${ }^{3}$ Sun Joon Bai, ${ }^{4}$ and Bae Hwan Lee ${ }^{1,2}$ \\ ${ }^{1}$ Department of Physiology, Yonsei University College of Medicine, Seoul 120-752, Republic of Korea \\ ${ }^{2}$ Brain Korea 21 PLUS Project for Medical Science, Yonsei University College of Medicine, Seoul 120-752, Republic of Korea \\ ${ }^{3}$ Division of Bio and Health Sciences, Mokwon University, Daejeon 302-729, Republic of Korea \\ ${ }^{4}$ Department of Anesthesiology and Pain Medicine, Yonsei University College of Medicine, Seoul 120-752, Republic of Korea \\ Correspondence should be addressed to Bae Hwan Lee; bhlee@yuhs.ac
}

Received 11 February 2015; Revised 16 April 2015; Accepted 17 April 2015

Academic Editor: Long-Jun Wu

Copyright (C) 2015 Jeongsoo Han et al. This is an open access article distributed under the Creative Commons Attribution License, which permits unrestricted use, distribution, and reproduction in any medium, provided the original work is properly cited.

\begin{abstract}
The insular cortex (IC) is associated with important functions linked with pain and emotions. According to recent reports, neural plasticity in the brain including the IC can be induced by nerve injury and may contribute to chronic pain. Continuous active kinase, protein kinase $\mathrm{M} \zeta(\mathrm{PKM} \zeta)$, has been known to maintain the long-term potentiation. This study was conducted to determine the role of PKM $\zeta$ in the IC, which may be involved in the modulation of neuropathic pain. Mechanical allodynia test and immunohistochemistry (IHC) of zif268, an activity-dependent transcription factor required for neuronal plasticity, were performed after nerve injury. After $\zeta$-pseudosubstrate inhibitory peptide (ZIP, a selective inhibitor of PKM $\zeta$ ) injection, mechanical allodynia test and immunoblotting of PKM $\zeta$, phospho-PKM (p-PKM $\zeta$ ), and GluR1 and GluR2 were observed. IHC demonstrated that zif268 expression significantly increased in the IC after nerve injury. Mechanical allodynia was significantly decreased by ZIP microinjection into the IC. The analgesic effect lasted for 12 hours. Moreover, the levels of GluR1, GluR2, and p-PKM $\zeta$ were decreased after ZIP microinjection. These results suggest that peripheral nerve injury induces neural plasticity related to PKM $\zeta$ and that ZIP has potential applications for relieving chronic pain.
\end{abstract}

\section{Introduction}

Nerve injury-induced neural plasticity in the central nervous system (CNS) is one of the important mechanisms involved in chronic pain [1-4]. Long-term potentiation (LTP), being considered as a neural substrate of learning and memory, is an underlying mechanism of synaptic plasticity $[5,6]$. Furthermore, several studies have reported that LTP is induced in the spinal cord and the cerebral cortex by acute and chronic pain states [7-9]. Thus, it is believed that there is a common mechanism between learning/memory and chronic pain [10].

Protein kinase $\mathrm{M} \zeta(\mathrm{PKM} \zeta)$ is a key molecule required for the maintenance of late LTP (L-LTP) [11, 12]. It is one of the atypical $\mathrm{PKC}$ isoforms, which include $\mathrm{PKM} \zeta, \mathrm{PKC} \zeta, \mathrm{PKC} \iota$, and PKC $\lambda$. Because PKM $\zeta$ has only the catalytic domain of
PKC $\zeta$, it can be activated persistently [13]. $\zeta$-pseudosubstrate inhibitory peptide (ZIP), a selective inhibitor of $\mathrm{PKM} \zeta$, can reverse LTP maintenance and block L-LTP induction $[14,15]$. Furthermore, ZIP can also reverse a variety of memory types, such as spatial information, fear, addiction, and conditioned taste aversion (CTA) memory [15-19]. Several studies have reported that $\mathrm{PKM} \zeta$-related pain memory can be erased in the spinal cord and the brain $[9,20,21]$.

The insular cortex (IC) is one of the pain-processing regions of the brain which is particularly related to the emotional aspects of pain [22-25]. Clinical and animal studies have shown that lesions of the IC cause pain asymbolia and can reverse the neuropathic pain state [26-28]. NMDA receptor-dependent plastic changes after nerve injury and $\mathrm{PKM} \zeta$-mediated CTA memory in the IC have been 
reported $[16,29]$. However, there have been no studies published on immediate-early gene (IEG) expression with respect to LTP and PKM $\zeta$-related mechanisms of plasticity in the IC after nerve injury.

Zif268, which is also known as early growth response protein 1 (EGR-1), is required for consolidation of L-LTP in the hippocampus and the spinal cord $[30,31]$. In order to determine whether there is a possibility of LTP induction in the IC after nerve injury, an immunohistochemical study on zif268 was conducted. Moreover, in order to reveal whether $\mathrm{PKM} \zeta$ signaling in the IC is involved in the maintenance of neuropathic pain, expression levels of $\mathrm{PKM} \zeta$ and phospho$\mathrm{PKM} \zeta$ ( $\mathrm{p}-\mathrm{PKM} \zeta$ ) were measured after ZIP injection into the IC, and analgesic effects following ZIP injection into the IC were observed.

\section{Materials and Methods}

2.1. Experimental Animals. Experimental protocols in this study complied with the National Institute of Health guidelines and were approved by the Institutional Animal Care and Use Committee of the Yonsei University Health System. Adult male Sprague-Dawley rats (Koatec, Pyeongtaek, Korea, 250$280 \mathrm{~g}$ ) were used. Rats were allowed to accommodate to the colony room for 7 days after arrival and were housed in plastic cages. Rats were maintained under a 12-hour light/dark cycle with food pellets and water provided ad libitum.

2.2. Cannulation and Neuropathic Surgery. For cannula implantation, rats were anesthetized with sodium pentobarbital (50 mg/kg, i.p). 28-gauge guide cannulas were bilaterally implanted into the IC (AP: $+1.0 \mathrm{~mm}, \mathrm{ML}: \pm 4.7 \mathrm{~mm}$, and DV: $-5.8 \mathrm{~mm}$ ) on a stereotaxic frame. Rats were allowed to recover for 7 days after cannula implantation. Neuropathic pain surgery was performed after recovery. Cannula-implanted rats were anesthetized with sodium pentobarbital $(50 \mathrm{mg} / \mathrm{kg}$, i.p.) and branches of the left sciatic nerve were exposed. The tibial and sural nerves were tightly ligated with 4-0 black silk and cut, while the common peroneal nerve was left intact [32]. The same procedure was applied to the sham-operated group without nerve injury. The wound was sutured in the muscle and skin layers.

2.3. Behavioral Test for Mechanical Allodynia. In order to observe the development of neuropathic pain, the mechanical allodynia test was performed by an experimenter blinded to the experiment before nerve injury, on postoperative days (PODs) 1 and 3. Rats were placed on a metal mesh floor under rectangular-shaped transparent domes. Animals were familiarized to the test conditions for 15 minutes before testing began. Withdrawal threshold was assessed by the application of electric von Frey filament (UGO Basile, Varese, Italy) stimulation to the sensitive area of the nerve-injured hind paw. This measurement was conducted 8 times at intervals of 2-3 minutes. The mechanical force was recorded when withdrawal responses of the hind paw occurred. Positive responses to mechanical stimulation included licking, sudden withdrawal, and biting of the ipsilateral paw.
2.4. ZIP Microinjection into the Insular Cortex and Analgesia Test. ZIP microinjection was conducted on POD 3. A Hamilton syringe and PE-10 tubing were used with an injection cannula for microinjection. Saline $(0.9 \% \mathrm{NaCl})$ or $10 \mathrm{nmol} / \mu \mathrm{L}$ of ZIP (Tocris Bioscience, Minneapolis, MN, USA) diluted in saline was infused into the IC bilaterally, $0.5 \mu \mathrm{L}$ per side. After injection, the injection cannulas were maintained in position as they were for at least 1 minute. The behavioral test was performed before ZIP injection and 30 minutes and 1, 2, 4, $8,12,24$, and 48 hours after microinjection.

2.5. Immunofluorescence Double Staining. For double immunofluorescence staining, rats were deeply anesthetized with urethane and perfused transcardially with saline followed by $4 \%$ paraformaldehyde in $0.1 \mathrm{M}$ sodium phosphate buffer $(\mathrm{PB}$, $\mathrm{pH}$ 6.8). The brain was removed from the skull and postfixed with $4 \%$ paraformaldehyde in $\mathrm{PB}$ at $4^{\circ} \mathrm{C}$ overnight. After postfixation, the brain block was transferred into phosphatebuffered saline (PBS, pH 7.4) containing 30\% sucrose for 24 hours. The brain sample was covered with a cryosection compound (frozen section compound FSC 22, Leica, Wetzlar, Germany) and was frozen in $-70^{\circ} \mathrm{C}$ deep freezer. Sample tissues were cut on a coronal section of $30 \mu \mathrm{m}$ thickness on a cryostat (HM 525, Thermo Scientific, Waltham, MA, USA). Section slides were then washed 3 times with $1 \mathrm{x}$ Tris-buffered saline (TBS) containing $0.025 \%$ Triton X-100. After washing, the section slides were incubated in $10 \%$ normal goat serum (Vector Laboratories, Burlingame, CA, USA) with 1\% bovine serum albumin (BSA, Thermo Scientific) in TBS for 1 hour at room temperature. The sections were incubated overnight in rabbit monoclonal anti-zif268 antibody $(1: 200$, Santa Cruz Biotechnology, Santa Cruz, CA, USA) and mouse monoclonal anti-NeuN antibody (1:200, Abcam, Cambridge, UK) diluted in $10 \%$ normal goat serum with TBS containing $1 \%$ BSA at $4^{\circ} \mathrm{C}$. The sections were then rinsed 2 times with $1 \mathrm{x}$ TBS plus 1\% Tween-20. After washing 2 times for 10 minutes, sections were incubated in secondary antibodies which were goat anti-rabbit Alexa Fluor 488 (1:200, Abcam) and goat anti-mouse Alexa Fluor 647 (1:200, Abcam), diluted in 10\% normal goat serum with TBS containing 1\% BSA for 1 hour at room temperature. Finally, the slides were washed 2 times with $1 x$ TBS containing $0.025 \%$ Triton X-100 and mounted in Vectashield mounting media (Vector Laboratories).

2.6. Immunohistochemistry. To calculate the population of zif268-positive cells, nickel-enhanced 3,3' -diaminobenzidine (DAB) immunostaining was performed on POD 3. Briefly, rat brain slices were prepared using the same steps as for immunofluorescence staining. For zif268 staining, section slides were rinsed 5 times with $1 \mathrm{x}$ PBS and incubated in methanol with $0.3 \% \mathrm{H}_{2} \mathrm{O}_{2}$ for 15 minutes to inhibit endogenous peroxidase activity. After washing the section slides 5 times, the sections were incubated with PBS containing $1 \%$ normal horse serum (Vector Laboratories) for 30 minutes and then incubated overnight at $4^{\circ} \mathrm{C}$ in $0.3 \%$ Triton $\mathrm{X}$ 100, 2\% normal horse serum (Vector Laboratories), and rabbit monoclonal anti-zif268 antibody $(1: 4,000$, Santa Cruz 
Biotechnology). The sections were rinsed 5 times with $1 x$ PBS and incubated for 30 minutes in a universal biotinylated antimouse/rabbit secondary antibody $(1: 50$, Vector Laboratories). Section slides were then washed again and incubated for 30 minutes with PBS containing avidin-biotinylated horseradish peroxidase complex (1:50, Vector Laboratories). Following washing 5 times for 15 minutes, sections were incubated for 5 minutes in a solution containing $0.1 \%$ of $\mathrm{DAB}$ and $0.1 \%$ ammonium nickel sulfate in $\mathrm{x}$ PBS and $0.01 \%$ $\mathrm{H}_{2} \mathrm{O}_{2}$. Finally, the sections were washed to stop the DAB reaction, serially dehydrated in 50,70, 95, and 100\% ethanol, cleared in xylene, and coverslipped with Permount (Fisher Scientific, Waltham, MA, USA).

To quantify zif268-positive cells in the IC, 8 representative sections of the IC were chosen from each brain. The interval of each section was $300 \mu \mathrm{m}$. Therefore, 8 sections cover anteroposterior range $(2.1 \mathrm{~mm})$ of the IC. Zif268-positive cells in the IC were identified with reference to the brain atlas of Paxinos and Watson [33]. All of the zif268-labeled cells from light-field microscopy image $(\times 20$ objective, Olympus BX40, Olympus, Tokyo, Japan) were counted manually. An experimenter blinded to the treatment conditions counted zif268-labeled cells of the ipsilateral and contralateral sides.

2.7. Western Blotting. To collect insular cortices, animals were anesthetized with enflurane and decapitated. The ipsilateral and contralateral rostral insular cortices were quickly isolated and transferred into a deep freezer. Extracted samples were stored at $-70^{\circ} \mathrm{C}$. For protein extraction, samples were homogenized by sonication in lysis buffer (Proprep, iNtRON Biotechnology Inc., Seongnam, Korea) containing phosphatase inhibitor (PhosStop, Roche, Penzberg, Germany). Samples were centrifuged at 22,250 g for 10 minutes at $4^{\circ} \mathrm{C}$ and supernatants were collected, and total protein concentrations of lysates were assessed with a spectrophotometer (ND1000, NanoDrop Technologies Inc., Wilmington, DE, USA). $10 \mu \mathrm{L}$ of protein of brain tissue extracts was denatured per well and run on $10 \%$ Bis-Tris gels (Bio-Rad, Hercules, CA, USA) for detection of PKM $\zeta$, p-PKM $\zeta$, GluR1, and GluR2. Proteins were transferred onto polyvinylidene difluoride (PVDF) membranes (GE Healthcare, Buckinghamshire, UK). Membranes were blocked in 5\% skim milk in TBS with Tween-20 for 1 hour and incubated in primary antibodies overnight on a rocking platform at $4^{\circ} \mathrm{C}$. Primary antibodies against PKM $\zeta$ $(1: 2,000$, Cell Signaling Technology, Beverly, MA, USA), p$\operatorname{PKM\zeta ~(1:2,000,~Cell~Signaling~Technology),~GluR1~}(1: 2,000$, Millipore, Temecula, MA, USA), GluR2 (1:2,000, Abcam), and GAPDH (1:10,000, Ab Frontier, Seoul, Korea), which was used as a loading control, were used for western blotting. On the following day, the membranes were incubated in the appropriate secondary antibodies for 2 hours and horseradish peroxidase activity was visualized using a chemiluminescent substrate (ECL Prime western blotting detection reagent, GE Healthcare) and processed with a local allocation system (LAS) (ImageQuant LAS 4000 Mini, GE Healthcare). The intensity of the bands for PKM $\zeta$, GluR1, and GluR2 was normalized to the intensity of GAPDH. The intensity of the bands for $\mathrm{p}-\mathrm{PKM} \zeta$ was normalized to the intensity of $\mathrm{PKM} \zeta$.
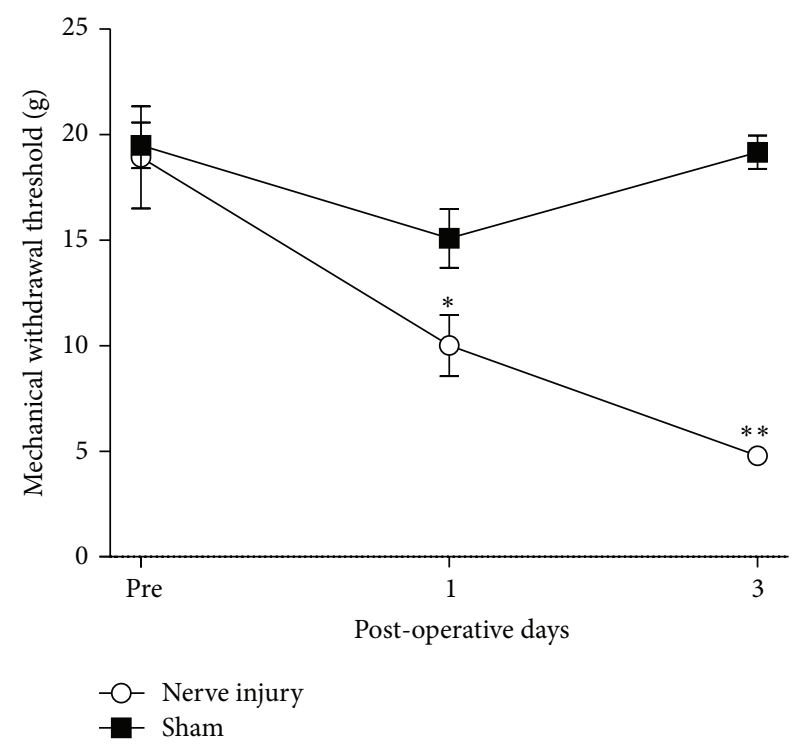

FIGURE 1: Development of mechanical allodynia after nerve injury. On PODs 1 and 3, rats developed significant neuropathic pain compared to sham group $\left({ }^{* *} P<0.01\right)$.

2.8. Statistical Analysis. Unpaired $t$-test for post hoc comparison was used for two-group comparisons while two-way ANOVA with repeated measures was used to analyze behavioral test. Western blots and IHC were compared using an unpaired $t$-test. All data were expressed as the mean \pm SEM. A $P$ value less than 0.05 was considered statistically significant.

\section{Results}

3.1. Development of Neuropathic Pain. Injury to two major branches (sural and tibial nerves) of the sciatic nerve induced mechanical allodynia on PODs 1 and 3 (Figure 1). Repeated measures two-way ANOVA indicated effects of group $\left(F_{1,13}=\right.$ 37.520, $P<0.001)$, PODs $\left(F_{2,26}=13.613, P<0.001\right)$, and interaction between group and PODs $\left(F_{2,26}=10.305\right.$, $P<0.01)$. The mechanical threshold of nerve-injured group decreased on POD 1 ( $n=8, P<0.01$, unpaired $t$-test $)$ and POD 3 ( $n=8, P<0.01$, unpaired $t$-test) relative to sham group $(n=8)$.

3.2. Immunofluorescence Double Labeling of Zif268 and NeuN. To confirm that zif268 was co-labeled with NeuN in the IC, double labeling of zif268 and NeuN was performed. The representative images of the nerve-injured group are shown in Figures 2(d), 2(e), and 2(f), and those of the sham group are shown in Figures 2(a), 2(b), and 2(c). Zif268 immunoreactivity (green) was observed in the IC (Figures 2(a) and 2(d)). NeuN, a neuronal marker (red), was observed in the IC (Figures 2(b) and 2(e)). Colocalization of zif268 (green) and NeuN (red) was detected in the IC (Figures 2(c) and 2(f)). As shown in Figure 2, zif268-positive cells were colocalized with NeuN-positive cells. This result indicates that zif268 is expressed in IC neurons. Nerve-injured rats have more zif268-positive cells (Figure 2(d)) than the sham 


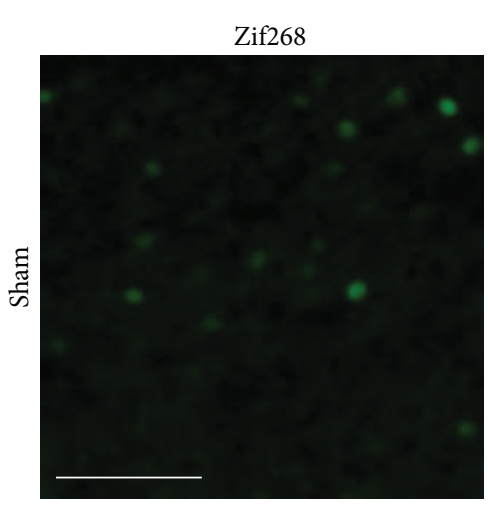

(a)

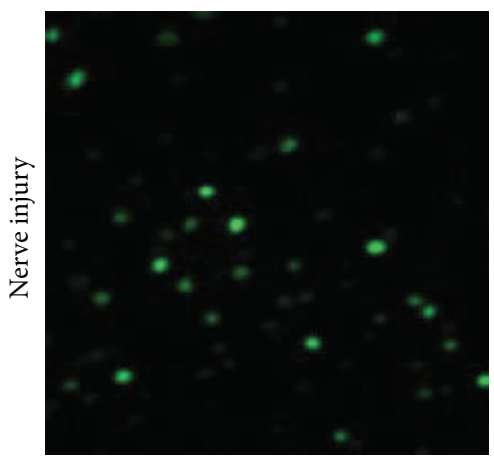

(d)

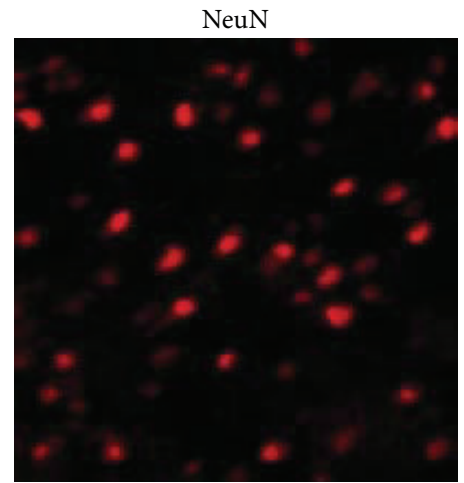

(b)

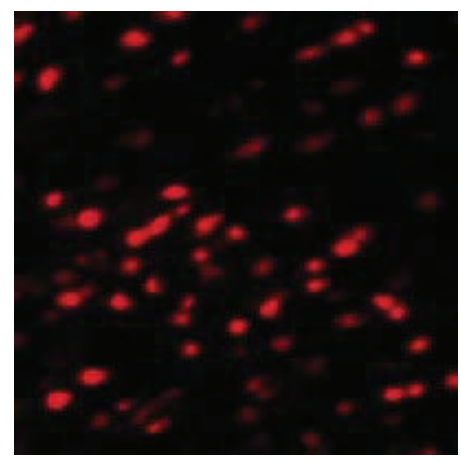

(e)

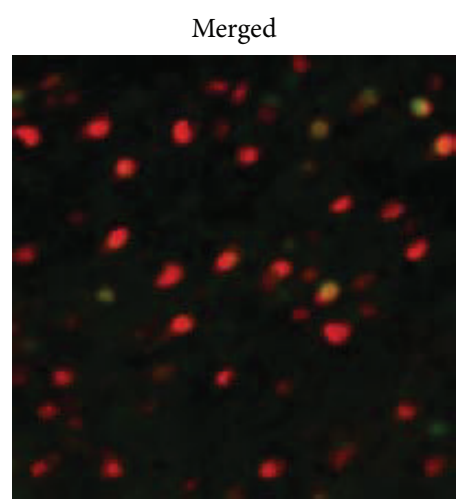

(c)

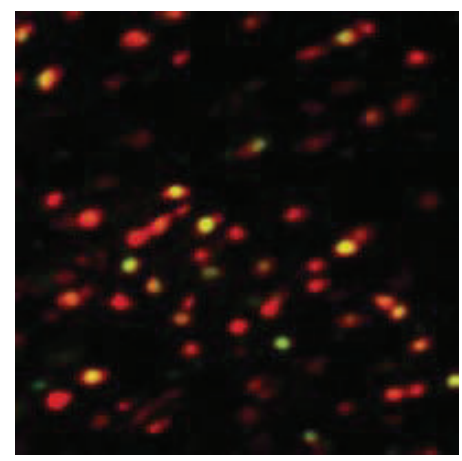

(f)

FIGURE 2: Fluorescence images of zif268 expression in the IC of nerve-injured and sham groups. (a) The sham group showed little expression of zif268-positive cells, unlike the nerve-injured group. (b) NeuN, a neuronal marker (red), was expressed in the sham group. (c) Colocalization of zif268 (green) and NeuN (red) is observed in the sham group. (d) In the nerve-injured group, the distribution of zif268 expression (green) was denser than in the sham group. (e) As in (b), NeuN was expressed in the nerve-injured group. (f) As in (c), colocalization of zif268 (green) and $\mathrm{NeuN}$ (red) is observed in the nerve-injured group. Scale bar, $50 \mu \mathrm{m}$.

group rats (Figure $2(\mathrm{a})$ ). The number of NeuN-positive cells in the IC is similar between the nerve-injured (Figure 2(e)) and sham-operated groups (Figure $2(\mathrm{~b})$ ). The merged data of zif268 and NeuN expression show that the IC has a relationship with neuropathic pain (Figures 2(c) and 2(f)).

\subsection{Immunohistochemistry of Zif268 in the Insular Cortex.} Zif268-positive cells were found in the IC of nerve-injured rats (Figures 3(a) and 3(b)). Furthermore, zif268 immunohistochemistry was performed to quantify the zif268-positive cells in the nerve-injured and sham groups. The results showed that the number of zif268-positive cells in the nerveinjured group was significantly increased compared to that of the sham group ( $n=6, P<0.05$, unpaired $t$-test; Figure 3(c)).

3.4. ZIP Injection into the Insular Cortex. Figure 4(a) shows the injection site of the IC. Injection of ZIP into the IC decreased mechanical allodynia gradually on POD 3. Repeated measures two-way ANOVA indicated effects of group $\left(F_{1,9}=11.798, P<0.01\right)$, time $\left(F_{7,63}=4.23, P<0.01\right)$, and interaction between group and time $\left(F_{7,63}=3.93, P<\right.$ $0.01)$. The time course of mechanical allodynia in the ZIPinjected group $(n=7)$ on POD 3 shows that the analgesic effects of ZIP last for 12 hours after injection $(P<0.05$, unpaired $t$-test; Figure 4(b)), where analgesia was measured relative to the saline-injected group $(n=5)$. However, at 24 and 48 hours after injection, ZIP had no significant effect $(P>0.05$, unpaired $t$-test).

3.5. Effects on PKM jection into the Insular Cortex. To determine the role of ZIP, the IC was punched 3 hours after ZIP injection. The total $\mathrm{PKM} \zeta$ in the ZIP-injected group $(n=6)$ was not changed $(P>0.05$, unpaired $t$-test; Figure 5(a)) on POD 3 relative to the saline-injected group $(n=6)$. However, the expression level of $\mathrm{p}-\mathrm{PKM} \zeta$ was downregulated by ZIP injection into the IC on POD $3(P<0.05$, unpaired $t$-test; Figure $5(\mathrm{~b}))$.

3.6. Effects of ZIP Microinjection into the Insular Cortex on GluR1 and GluR2 Levels. We speculated that the effects of ZIP may contribute to inhibition of AMPA receptors. Accordingly, the expression levels of the AMPA receptor subunits GluR1 and GluR2 were measured after ZIP microinjection on POD 3. The results showed decreased GluR1 and GluR2 levels $(P<0.05$, unpaired $t$-test; Figures 6(a) and 6(b), resp. $)$ in the ZIP-injected group $(n=8)$, relative to the saline-injected group $(n=8)$. 

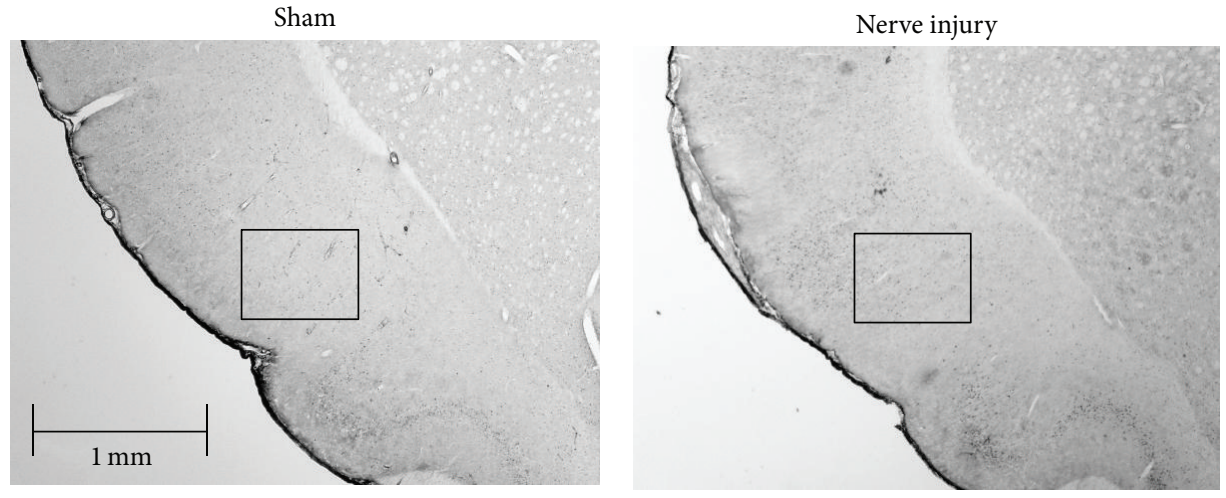

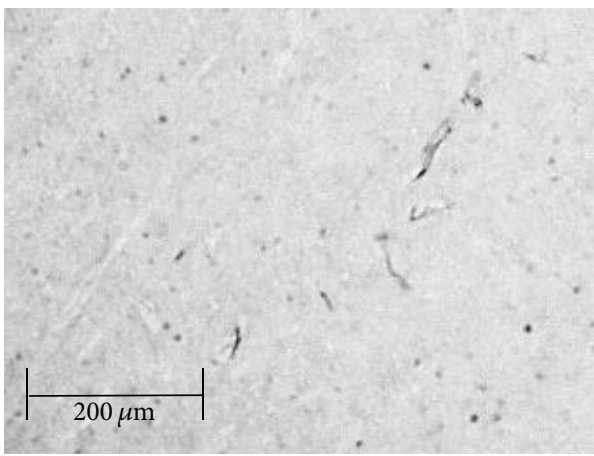

(a)

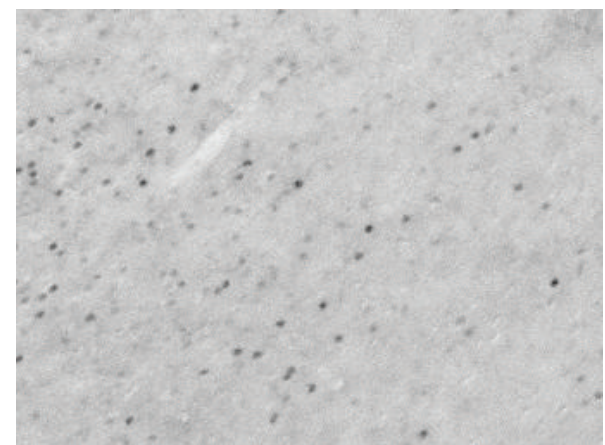

(b)

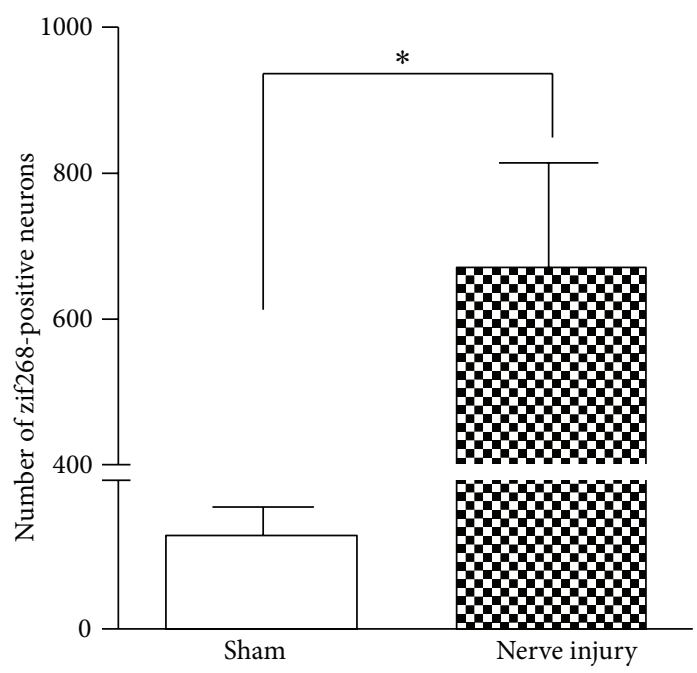

(c)

FIgURE 3: Zif268-positive cells in the IC. (a) Zif268-positive cells in the IC of a sham-operated rat. (b) Zif268-positive cells in the IC of a nerve-injured rat. (c) Comparison of zif268-positive cells in the nerve-injured and sham groups. Zif268-positive cells increased significantly in the IC after nerve injury $\left({ }^{*} P<0.05\right)$. Cell counts are expressed per section.

\section{Discussion}

The IC plays a role in interpretation of emotional aspects of pain as one of the limbic system areas [22]. Several studies have reported that plastic changes in the IC are induced after peripheral nerve injury $[29,34]$. Therefore, we focused on plastic changes of the IC and its pain modulation after nerve injury in order to understand the mechanisms of chronic pain. Recent reports have shown that functional changes in the brain following nerve injury may be mediated by LTP $[2$, 9]. PKM $\zeta$ plays a key role in maintaining LTP, and inhibition of PKM $\zeta$ can reverse the chronic pain state and can erase established memories [12,18]. Accordingly, the present study was performed to determine the plastic changes related to LTP formation, maintenance after nerve injury, and the role of the IC in pain modulation. Our findings suggest that PKM $\zeta$ in the IC can lead to nerve injury-induced plasticity which contributes to the maintenance of neuropathic pain states. 


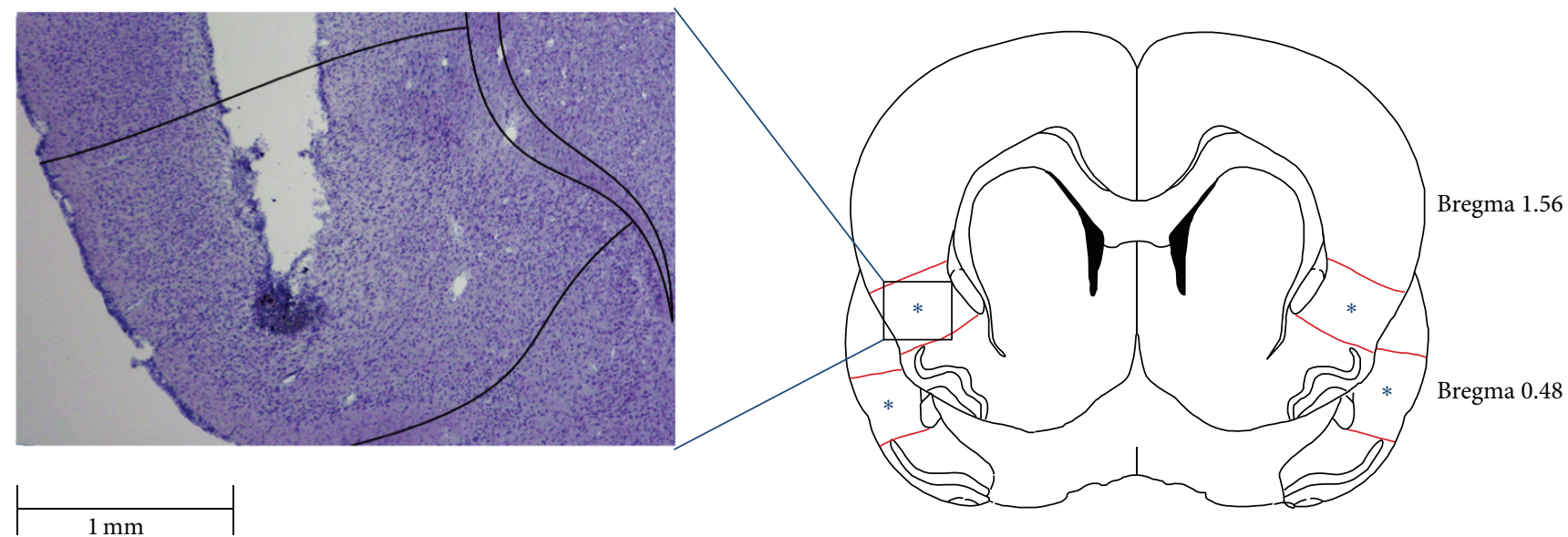

(a)

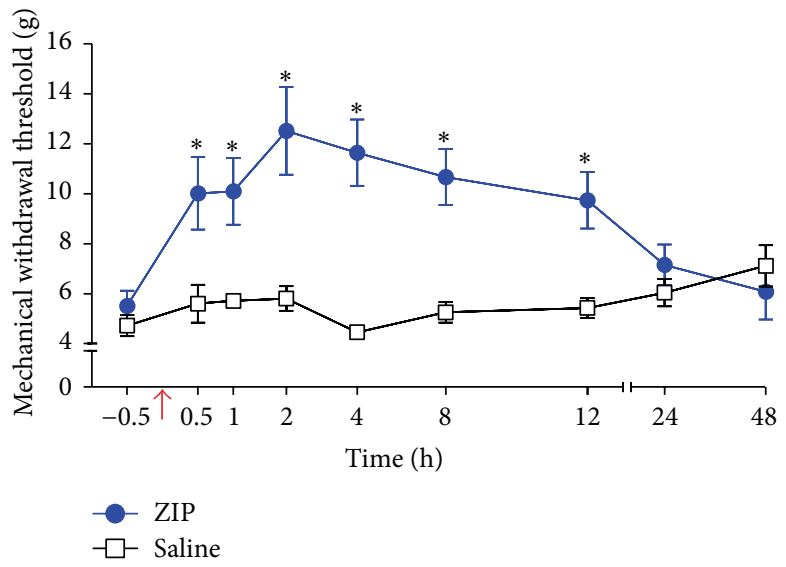

(b)

FIGURE 4: Reduction of mechanical allodynia after ZIP microinjection into the IC. (a) Identification of the ZIP injection site. ZIP was microinjected into the IC. (b) Paw withdrawal threshold to mechanical stimulation in POD 3 rats after microinjection of ZIP. Significant differences between nerve-injured and sham groups were found at the time points from 30 minutes to 12 hours after injection $\left({ }^{*} P<0.05\right)$. The arrow indicates the time point of ZIP or saline injection.

Zif268 is a well-known marker related to LTP and neuronal plasticity [30]. In the hippocampus, the L-LTP phase requires the expression of IEGs such as zif268 and activityregulated cytoskeletal-associated protein (Arc) $[35,36]$. LTPinducing stimulation increases zif268 expression levels in the hippocampus [37]. Furthermore, zif268 knockout mice displayed an absence of L-LTP and impaired long-term memory [30]. c-Fos is a neuronal activation marker and is expressed highly after nerve injury in the spinal cord and anterior cingulate cortex (ACC) [9]. Expression of c-Fos in the IC is increased by stress-induced hyperalgesia [38]. However, since zif268 is more correlated with LTP and plasticity to a greater degree than is c-Fos [39], changes in zif268 expression level in the IC after nerve injury were assessed immunohistochemically in our study. There have been several reports that zif268 is induced by nociceptive stimulation $[39,40]$. However, there have been no reports on zif268 expression related to nerve injury-induced plasticity in the IC. Our results show that the number of zif268-positive cells increased after nerve injury, which is related to nerve injury-induced LTP in the IC.
Animals with lesions of the IC show reversed neuropathic or inflammatory pain behavior $[27,28]$. Patients with lesions in the IC did not display appropriate responses to pain, but all other sensory and cognitive functions still remained intact [26]. Moreover, painful stimuli activate the IC, and direct stimulation of the IC can evoke sensations of pain, indicating that the IC is pronociceptive and involved in the interpretation of pain sensation $[23,41,42]$.

$\mathrm{PKM} \zeta$ is a key molecule for maintaining L-LTP and inhibition of PKM $\zeta$ can erase established LTP $[12,18]$. Although the functions of PKM $\zeta$ were reported to be controversial in some studies [43-45], recent studies have suggested that atypical PKCs compensate the PKM $\zeta$ role of maintaining LTP in the constitutive PKM $\zeta$ knockout mice [46]. In fact, many studies have reported that the administration of ZIP into the spinal cord can reverse inflammatory pain, but not neuropathic pain $[20,21,47,48]$. In contrast, ZIP injection into the ACC reverses the neuropathic pain state, but the analgesic effects disappear 24 hours after injection of ZIP [9]. Based on this report, the effects of ZIP injection into the IC were 

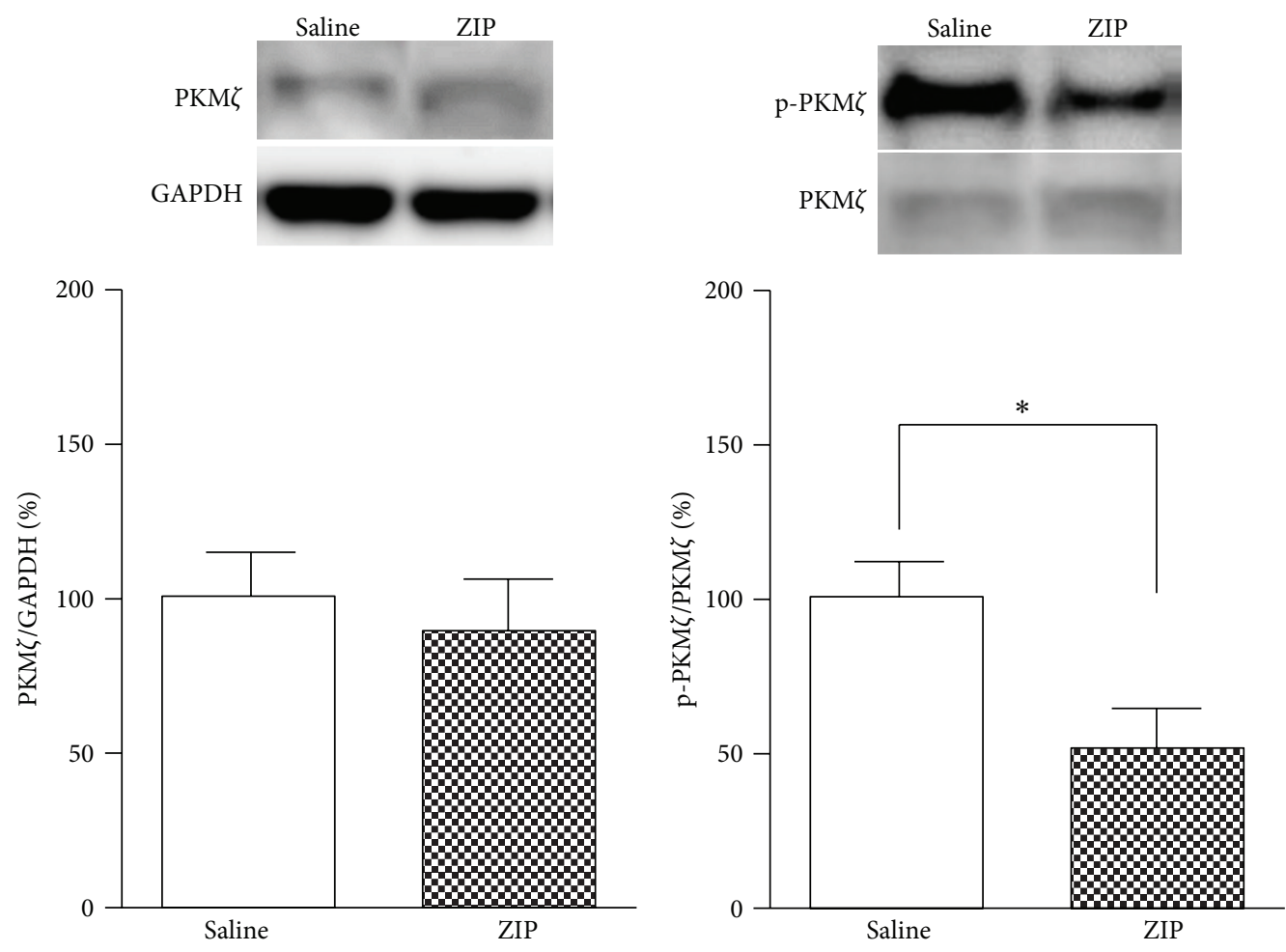

(a)

(b)

FIGURE 5: Expression levels of PKM $\zeta$ and p-PKM $\zeta$ after ZIP microinjection into the IC on POD 3. (a) Expression levels of PKM $\zeta$ normalized to GAPDH levels. There was no significant difference in PKM $\zeta$ levels between the saline injection and ZIP injection groups $(P>0.05)$. (b) Expression levels of p-PKM $\zeta$ normalized to PKM $\zeta$ levels. After ZIP injection, p-PKM $\zeta$ levels were significantly decreased $\left({ }^{*} P<0.05\right)$.

investigated in the present study. The effects of ZIP lasted for 12 hours after injection into the IC. Our findings suggest that nerve injury induces plasticity related to $\mathrm{PKM} \zeta$ in the IC and that the IC has a pain modulation function. Interestingly, the pain-relieving effect was not permanent. This phenomenon has been observed in other reports utilizing neuropathic pain models. This may be due to the reestablishment of LTP by the tonic peripheral afferent drive $[9,48]$.

$\mathrm{PKM} \zeta$ is selectively upregulated in the spinal cord by formalin, capsaicin, and nerve injury $[21,48]$. In the ACC, both $\mathrm{PKM} \zeta$ and $\mathrm{p}-\mathrm{PKM} \zeta$ increased after nerve injury [9]. $\mathrm{PKM} \zeta$ is activated by phosphorylation and can be inhibited by ZIP [18]. Increased levels of p-PKM $\zeta$ in the ACC contribute to neuropathic pain [9]. Similarly, increased levels of $\mathrm{p}-\mathrm{PKM} \zeta$ in the spinal cord contribute to formalin-induced pain [47]. Taken together, we hypothesized that upregulation of $\mathrm{p}-\mathrm{PKM} \zeta$ might contribute to neuropathic pain states and that ZIP can alleviate neuropathic pain. Indeed, decreased levels of $\mathrm{p}-\mathrm{PKM} \zeta$ were observed following ZIP injection. However, the expression levels of $\mathrm{PKM} \zeta$ did not change after ZIP injection. In contrast, intrathecal infusion of ZIP did not reduce $\mathrm{p}-\mathrm{PKM} \zeta$ levels in the spinal cords of formalin model rats [47]. Although our findings are inconsistent with these studies, our results show that the function of $\mathrm{p}-\mathrm{PKM} \zeta$ in the IC differs from that in the spinal cord, and these results are in line with those found with respect to the ACC [9].

Several reports have suggested that trafficking of the GluR2 subunit of the AMPA receptor in the hippocampus is related to $\mathrm{PKM} \zeta[12,49]$. In addition, fear memory is maintained by $\mathrm{PKM} \zeta$-mediated GluR2-dependent AMPA receptor trafficking in the amygdala [50]. Other reports suggest that the GluR1 subunit of the AMPA receptor is involved in the molecular machinery of cocaine-induced plasticity in the nucleus accumbens (NAc) $[51,52]$. PKM $\zeta$-mediated LTP expression may be induced by $\mathrm{N}$-ethylmaleimide-sensitive factor/GluR2-dependent trafficking in the hippocampus [49]. Interestingly, our results show that both GluR1 and GluR2 levels are downregulated by ZIP injection in the neuropathic pain model rats. Consistent with this, GluR1 levels were decreased after ZIP injection into the ACC of neuropathic pain model [9]. Other reports suggest that activation of metabotropic GluR1 (mGluR1) is required for insular L-LTP induction [53]. In addition, administration of ZIP into the NAc core can abolish long-term drug reward memory by effect on GluR2-containing AMPA receptors [54]. Taken together, we assume that $\mathrm{PKM} \zeta$ maintains pain-related longterm plastic changes in the IC via both the GluR1 and GluR2 subunits of AMPA receptors. 


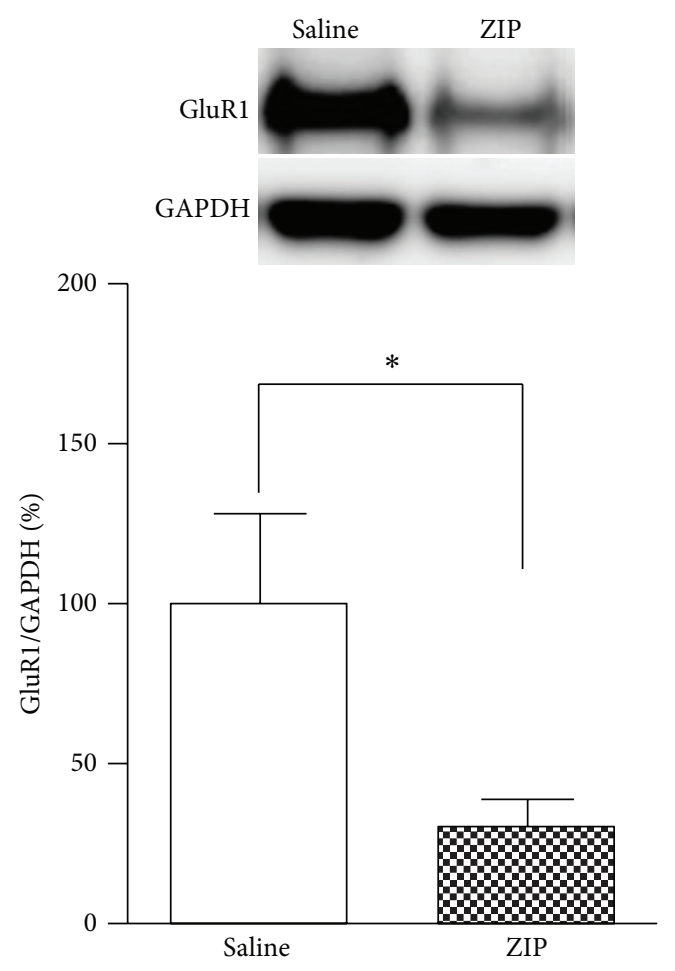

(a)

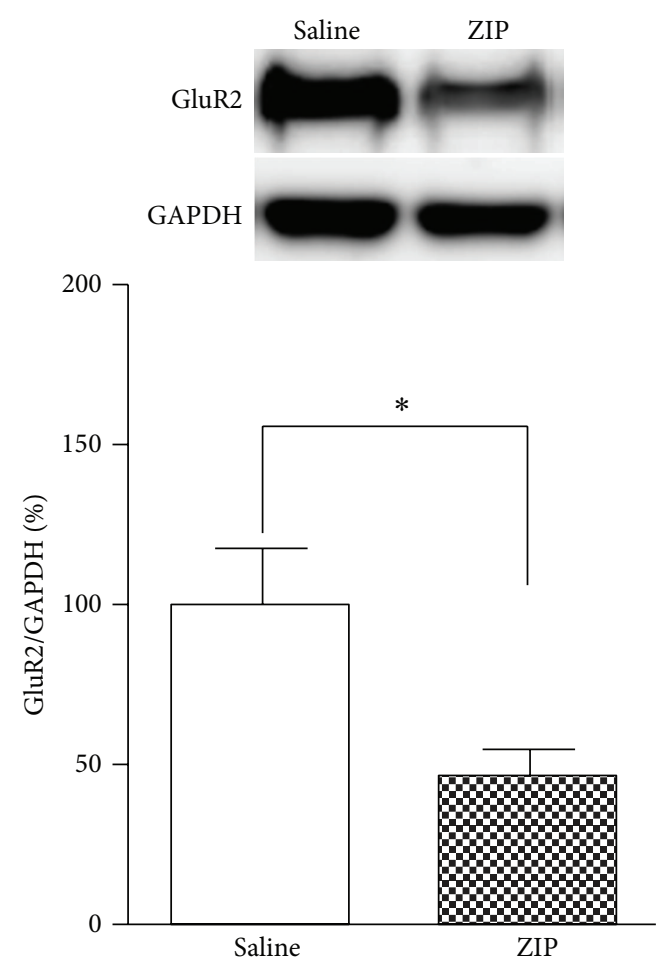

(b)

FIGURE 6: Expression levels of GluR1 and GluR2 after ZIP microinjection into the IC on POD 3. (a) Expression levels of GluR1 normalized to GAPDH levels. There was a significant difference between GluR1 levels in the saline injection and ZIP injection groups. After microinjection of ZIP, levels of GluR1 were significantly decreased $\left({ }^{*} P<0.05\right)$. (b) Expression levels of GluR2 normalized to GAPDH levels. After ZIP injection, GluR2 levels were significantly decreased $\left({ }^{*} P<0.05\right)$.

\section{Conclusion}

In this study, we demonstrated that there is a correlation between PKM $\zeta$ and neural plasticity of the IC, which might explain chronic pain mechanisms. PKM $\zeta$ seems to mediate this plasticity by regulating AMPA receptors containing GluR1 and GluR2. Moreover, the pain modulation function of the IC related to $\mathrm{PKM} \zeta$ was revealed through administration of ZIP. Pharmacological targeting to inhibit plasticity of the IC may therefore present a strategy for chronic neuropathic pain therapy.

\section{Conflict of Interests}

The authors declare that there is no conflict of interests regarding the publication of this paper.

\section{Authors' Contribution}

Jeongsoo Han and Minjee Kwon contributed equally to this work.

\section{Acknowledgment}

This work was supported by the National Research Foundation of Korea founded by the Ministry of Science, ICT and Future Planning (NRF-2014R1A2A2A04004407).

\section{References}

[1] R. Suzuki and A. Dickenson, "Spinal and supraspinal contributions to central sensitization in peripheral neuropathy," NeuroSignals, vol. 14, no. 4, pp. 175-181, 2005.

[2] M. Zhuo, "Cortical excitation and chronic pain," Trends in Neurosciences, vol. 31, no. 4, pp. 199-207, 2008.

[3] R. Ruscheweyh, O. Wilder-Smith, R. Drdla, X.-G. Liu, and J. Sandkühler, "Long-term potentiation in spinal nociceptive pathways as a novel target for pain therapy," Molecular Pain, vol. 7, article 20, 2011.

[4] C. J. Woolf and M. W. Salter, "Neuronal plasticity: increasing the gain in pain," Science, vol. 288, no. 5472, pp. 1765-1768, 2000.

[5] T. V. P. Bliss and G. L. Collingridge, "A synaptic model of memory: long-term potentiation in the hippocampus," Nature, vol. 361, no. 6407, pp. 31-39, 1993.

[6] S. F. Cooke and T. V. P. Bliss, "Plasticity in the human central nervous system," Brain, vol. 129, no. 7, pp. 1659-1673, 2006.

[7] J. Sandkühler and X. Liu, "Induction of long-term potentiation at spinal synapses by noxious stimulation or nerve injury," European Journal of Neuroscience, vol. 10, no. 7, pp. 2476-2480, 1998.

[8] H. Ikeda, J. Stark, H. Fischer et al., "Synaptic amplifier of inflammatory pain in the spinal dorsal horn," Science, vol. 312, no. 5780, pp. 1659-1662, 2006.

[9] X. Y. Li, H. G. Ko, T. Chen et al., "Alleviating neuropathic pain hypersensitivity by inhibiting PKMzeta in the anterior cingulate cortex," Science, vol. 330, no. 6009, pp. 1400-1404, 2010. 
[10] R. R. Ji, T. Kohno, K. A. Moore, and C. J. Woolf, "Central sensitization and LTP: do pain and memory share similar mechanisms?" Trends in Neurosciences, vol. 26, no. 12, pp. 696705, 2003.

[11] D. S. F. Ling, L. S. Benardo, P. A. Serrano et al., "Protein kinase Mzeta is necessary and sufficient for LTP maintenance," Nature Neuroscience, vol. 5, no. 4, pp. 295-296, 2002.

[12] T. C. Sacktor, "PKMzeta, LTP maintenance, and the dynamic molecular biology of memory storage," Progress in Brain Research, vol. 169, pp. 27-40, 2008.

[13] T. C. Sacktor, P. Osten, H. Valsamis, X. Jiang, M. U. Naik, and E. Sublette, "Persistent activation of the $\zeta$ isoform of protein kinase $\mathrm{C}$ in the maintenance of long-term potentiation," Proceedings of the National Academy of Sciences of the United States of America, vol. 90, no. 18, pp. 8342-8346, 1993.

[14] P. Serrano, Y. Yao, and T. C. Sacktor, "Persistent phosphorylation by protein kinase Mzeta maintains late-phase long-term potentiation," The Journal of Neuroscience, vol. 25, no. 8, pp. 19791984, 2005.

[15] E. Pastalkova, P. Serrano, D. Pinkhasova, E. Wallace, A. A. Fenton, and T. C. Sacktor, "Storage of spatial information by the maintenance mechanism of LTP," Science, vol. 313, no. 5790, pp. 1141-1444, 2006

[16] R. Shema, T. C. Sacktor, and Y. Dudai, "Rapid erasure of longterm memory associations in the cortex by an inhibitor of

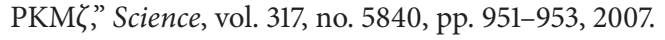

[17] P. Serrano, E. L. Friedman, J. Kenney et al., "РКM $\zeta$ maintains spatial, instrumental, and classically conditioned long-term memories," PLoS Biology, vol. 6, no. 12, article e318, 2008.

[18] T. J. Price and S. Ghosh, "ZIPping to pain relief: the role (or not) of PKM $\zeta$ in chronic pain," Molecular Pain, vol. 9, no. 1, article 6, 2013.

[19] M. E. Vélez-Hernández, R. Vázquez-Torres, M. C. VelasquezMartinez et al., "Inhibition of Protein kinase Mzeta (PKMzeta) in the mesolimbic system alters cocaine sensitization in rats," Journal of Drug and Alcohol Research, vol. 2, Article ID 235669, 12 pages, 2013.

[20] M. N. Asiedu, D. V. Tillu, O. K. Melemedjian et al., "Spinal protein kinase $\mathrm{M}$ zeta underlies the maintenance mechanism of persistent nociceptive sensitization," The Journal of Neuroscience, vol. 31, no. 18, pp. 6646-6653, 2011.

[21] A. Laferrière, M. H. Pitcher, A. Haldane et al., "PKM $\zeta$ is essential for spinal plasticity underlying the maintenance of persistent pain," Molecular Pain, vol. 7, article 99, 2011.

[22] A. D. Craig, "Interoception: the sense of the physiological condition of the body," Current Opinion in Neurobiology, vol. 13, no. 4, pp. 500-505, 2003.

[23] L. Jasmin, S. D. Rabkin, A. Granato, A. Boudah, and P. T. Ohara, "Analgesia and hyperalgesia from GABA-mediated modulation of the cerebral cortex," Nature, vol. 424, no. 6946, pp. 316-320, 2003.

[24] L. Jasmin, A. R. Burkey, A. Granato, and P. T. Ohara, "Rostral agranular insular cortex and pain areas of the central nervous system: a tract-tracing study in the rat," The Journal of Comparative Neurology, vol. 468, no. 3, pp. 425-440, 2004.

[25] P. Alvarez, W. Dieb, A. Hafidi, D. L. Voisin, and R. Dallel, "Insular cortex representation of dynamic mechanical allodynia in trigeminal neuropathic rats," Neurobiology of Disease, vol. 33, no. 1, pp. 89-95, 2009.

[26] M. Berthier, S. Starkstein, and R. Leiguarda, "Asymbolia for pain: a sensory-limbic disconnection syndrome," Annals of Neurology, vol. 24, no. 1, pp. 41-49, 1988.
[27] A. M. Benison, S. Chumachenko, J. A. Harrison et al., "Caudal granular insular cortex is sufficient and necessary for the longterm maintenance of allodynic behavior in the rat attributable to mononeuropathy," The Journal of Neuroscience, vol. 31, no. 17, pp. 6317-6328, 2011.

[28] U. Coffeen, J. Manuel Ortega-Legaspi, F. J. López-Muñoz, K. Simón-Arceo, O. Jaimes, and F. Pellicer, "Insular cortex lesion diminishes neuropathic and inflammatory pain-like behaviours," European Journal of Pain, vol. 15, no. 2, pp. 132-138, 2011.

[29] S. Qiu, T. Chen, K. Koga et al., "An increase in synaptic NMDA receptors in the insular cortex contributes to neuropathic pain," Science Signaling, vol. 6, no. 275, article ra34, 2013.

[30] M. W. Jones, M. L. Errington, P. J. French et al., "A requirement for the immediate early gene Zif268 in the expression of late LTP and long-term memories," Nature Neuroscience, vol. 4, no. 3, pp. 289-296, 2001.

[31] F. Haugan, K. Wibrand, A. Fiskå, C. R. Bramham, and A. Tjølsen, "Stability of long term facilitation and expression of zif268 and Arc in the spinal cord dorsal horn is modulated by conditioning stimulation within the physiological frequency range of primary afferent fibers," Neuroscience, vol. 154, no. 4, pp. 1568-1575, 2008.

[32] B. H. Lee, R. Won, E. J. Baik, S. H. Lee, and C. H. Moon, "An animal model of neuropathic pain employing injury to the sciatic nerve branches," NeuroReport, vol. 11, no. 4, pp. 657-661, 2000.

[33] G. Paxinos and C. Watson, The Rat Brain in Stereotaxic Coordinates, Academic Press/Elsevier, Boston, Mass, USA, 6th edition, 2007.

[34] S. Qiu, M. Zhang, Y. Liu et al., "GluA1 phosphorylation contributes to postsynaptic amplification of neuropathic pain in the insular cortex," The Journal of Neuroscience, vol. 34, no. 40, pp. 13505-13515, 2014.

[35] J. D. Shepherd and M. F. Bear, "New views of Arc, a master regulator of synaptic plasticity," Nature Neuroscience, vol. 14, no. 3, pp. 279-284, 2011.

[36] N. Granado, O. Ortiz, L. M. Suárez et al., " $D_{1}$ but not $D_{5}$ dopamine receptors are critical for LTP, spatial learning, and LTP-induced arc and zif268 expression in the hippocampus," Cerebral Cortex, vol. 18, no. 1, pp. 1-12, 2008.

[37] F. Ranieri, M. V. Podda, E. Riccardi et al., "Modulation of LTP at rat hippocampal CA3-CA1 synapses by direct current stimulation," Journal of Neurophysiology, vol. 107, no. 7, pp. 18681880, 2012.

[38] R. Takeda, Y. Watanabe, T. Ikeda et al., "Analgesic effect of milnacipran is associated with c-Fos expression in the anterior cingulate cortex in the rat neuropathic pain model," Neuroscience Research, vol. 64, no. 4, pp. 380-384, 2009.

[39] W. Wisden, M. L. Errington, S. Williams et al., "Differential expression of immediate early genes in the hippocampus and spinal cord," Neuron, vol. 4, no. 4, pp. 603-614, 1990.

[40] L. J. Rygh, R. Suzuki, W. Rahman et al., "Local and descending circuits regulate long-term potentiation and zif268 expression in spinal neurons," The European Journal of Neuroscience, vol. 24, no. 3, pp. 761-772, 2006.

[41] L. Mazzola, J. Isnard, R. Peyron, M. Guénot, and F. Mauguière, "Somatotopic organization of pain responses to direct electrical stimulation of the human insular cortex," Pain, vol. 146, no. 1-2, pp. 99-104, 2009. 
[42] M. Frot, M. Magnin, F. Mauguière, and L. Garcia-Larrea, "Human SII and posterior insula differently encode thermal laser stimuli," Cerebral Cortex, vol. 17, no. 3, pp. 610-620, 2007.

[43] A. M. Lee, B. R. Kanter, D. Wang et al., "Prkcz null mice show normal learning and memory," Nature, vol. 493, no. 7432, pp. 416-419, 2013.

[44] L. J. Volk, J. L. Bachman, R. Johnson, Y. Yu, and R. L. Huganir, "PKM- $\zeta$ is not required for hippocampal synaptic plasticity, learning and memory," Nature, vol. 493, no. 7432, pp. 420-423, 2013.

[45] A. X. Wu-Zhang, C. L. Schramm, S. Nabavi, R. Malinow, and A. C. Newton, "Cellular pharmacology of protein kinase $M \zeta$ (PKM $\zeta)$ contrasts with its in vitro profile: implications for PKM $\zeta$ as a mediator of memory," The Journal of Biological Chemistry, vol. 287, no. 16, pp. 12879-12885, 2012.

[46] Y. Yao, P. Tsokas, D. Jothianandan, A. Tcherepanov, P. van de Nes, and T. C. Sacktor, "Compensation by $\mathrm{PKC} \iota / \lambda$ activation and $\mathrm{PKM} / / \lambda$ formation in PKM $\zeta$ knock-out mice," Society for Neuroscience Abstract, no. 233.22, 2013, http://www.abstractsonline.com/Plan/ViewAbstract.aspx?mID=3236\&sKey=6bc2b62bcc81-4ad4-9245-52909d0cf884\&cKey=ff4334ac-acb0-4745-8559d4fd4la58ed1\&mKey=8d2a5bec-4825-4cd6-9439-b42bb151dlcf.

[47] F. Marchand, R. D’Mello, P. K. Yip et al., "Specific involvement of atypical PKCzeta/PKMzeta in spinal persistent nociceptive processing following peripheral inflammation in rat," Molecular Pain, vol. 7, article 86, 2011.

[48] T. King, C. Qu, A. Okun et al., "Contribution of PKM $\zeta$ dependent and independent amplification to components of experimental neuropathic pain," Pain, vol. 153, no. 6, pp. 12631273, 2012.

[49] Y. Yao, M. T. Kelly, S. Sajikumar et al., "PKM $\zeta$ maintains late long-term potentiation by $N$-ethylmaleimide-sensitive factor/GluR2-dependent trafficking of postsynaptic AMPA receptors," The Journal of Neuroscience, vol. 28, no. 31, pp. 78207827, 2008.

[50] P. V. Migues, O. Hardt, D. C. Wu et al., "РКM $\zeta$ maintains memories by regulating GluR2-dependent AMPA receptor trafficking," Nature Neuroscience, vol. 13, no. 5, pp. 630-634, 2010.

[51] D. Shabashov, E. Shohami, and R. Yaka, "Inactivation of PKM $\zeta$ in the NAc shell abolished cocaine-conditioned reward," Journal of Molecular Neuroscience, vol. 47, no. 3, pp. 546-553, 2012.

[52] M. A. Sutton, E. F. Schmidt, K.-H. Choi et al., "Extinctioninduced upregulation in AMPA receptors reduces cocaineseeking behaviour," Nature, vol. 421, no. 6918, pp. 70-75, 2003.

[53] M.-G. Liu, S. J. Kang, T.-Y. Shi et al., "Long-term potentiation of synaptic transmission in the adult mouse insular cortex: multielectrode array recordings," Journal of Neurophysiology, vol. 110, no. 2, pp. 505-521, 2013.

[54] Y.-Q. Li, Y.-X. Xue, Y.-Y. He et al., "Inhibition of PKM in nucleus accumbens core abolishes long-term drug reward memory," Journal of Neuroscience, vol. 31, no. 14, pp. 5436-5446, 2011. 

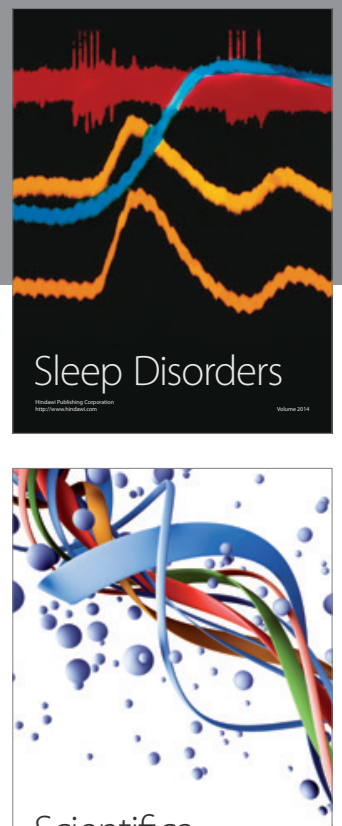

Scientifica
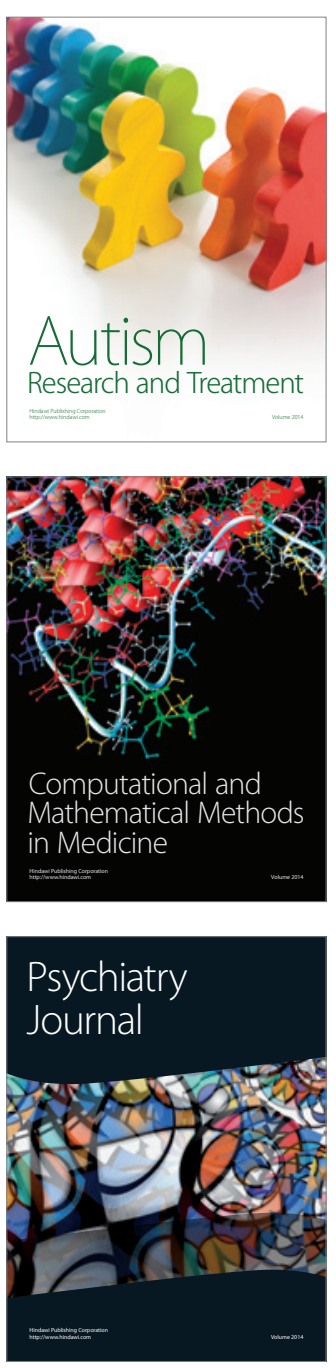
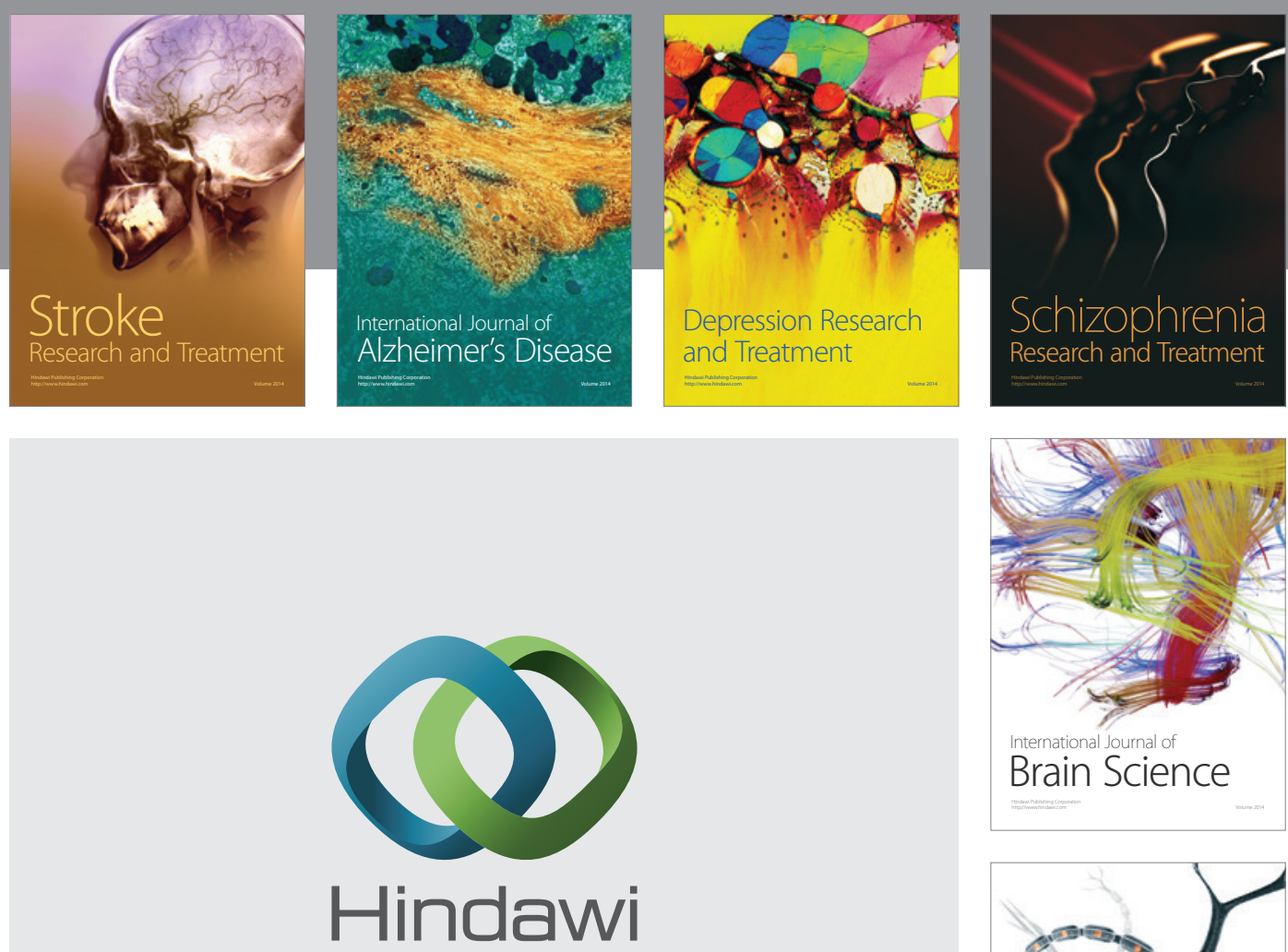

Submit your manuscripts at

http://www.hindawi.com
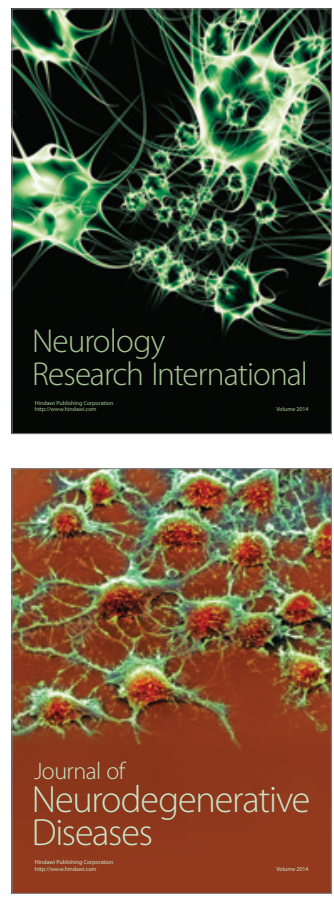

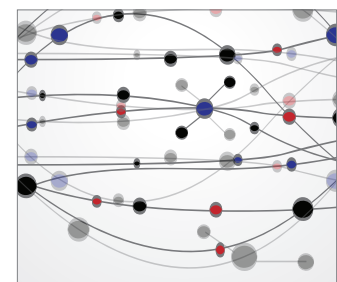

The Scientific World Journal
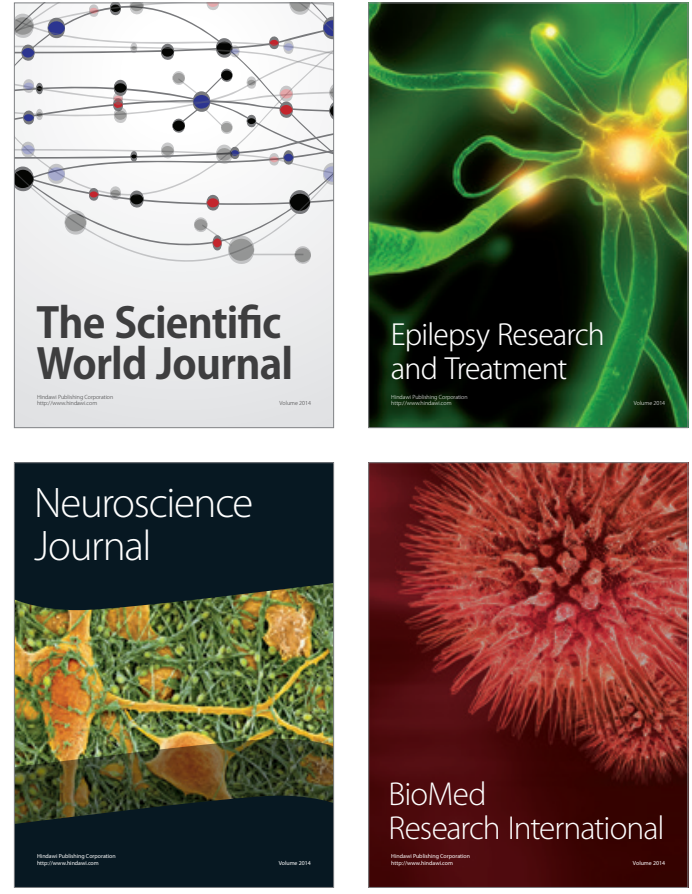

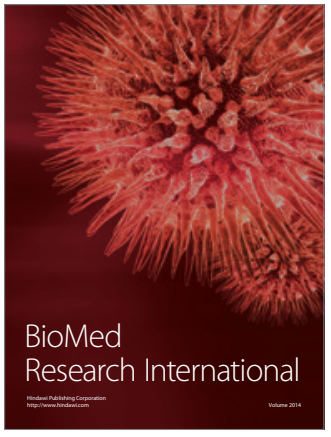

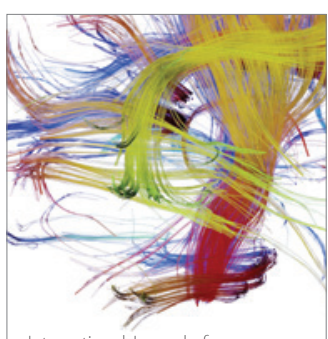

Brain Science

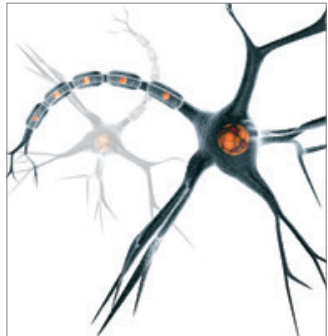

Neural Plasticity
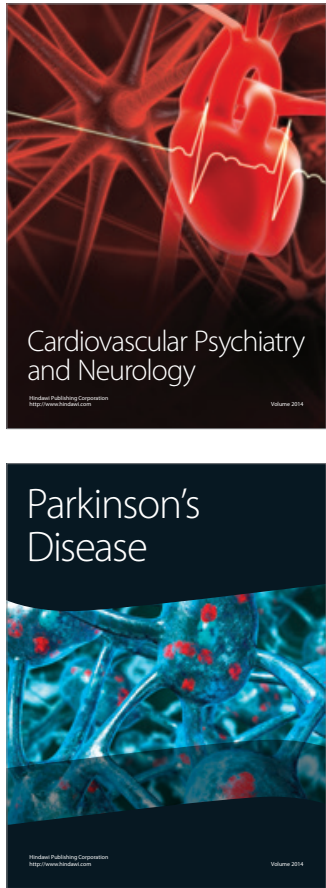